\title{
Usual and Unusual Case of Foreign Body in the Bronchus
}

\author{
Dhanasekar T., Amarabalan Rajendran, Krishna Rao U., Prasanna Kumar Thomas \\ Department of Respiratory medicine, Sri Ramachandra University, Chennai, Tamilnadu, India.
}

\section{A B STRACT}

Tracheobronchial foreign body (TFB) aspiration can be a life-threatening emergency associated with significant morbidity and mortality. Aspiration of foreign bodies into the tracheobronchial tree is common in pediatric age group but rarer in adults. Adults account for only about $20 \%$ of the reported cases. The clinical presentation in adults may vary from being asymptomatic to severe respiratory distress. In this case report, we describe two cases of bronchial foreign body one with an unusual presentation of a common foreign body and the other an unusual foreign body.

Key words: Tracheobronchial foreign body, Bronchoscopy, Central obstructive collapse, Intubation Stylet

\section{INTRODUCTION}

Aspiration of foreign bodies into tracheo-bronchial tree is infrequently seen in adults. ${ }^{1}$ Incidence rates increase with advancing age. Risk factors of foreign body aspiration in adults include advanced age, altered state of consciousness due to alcohol or drugs, poor dentition and neurological disorders such as Parkinson's disease and stroke. ${ }^{2}$ The foreign bodies can be dietary or nondietary but are associated with similar sequelae. ${ }^{3}$ Potential complications of an aspirated foreign body include post obstructive pneumonia, lung abscess formation, bronchiectasis, foreign body granuloma and central obstructive collapse. ${ }^{4}$ Occult foreign bodies can remain undetected for months to years and often are misdiagnosed. Therefore a high index of suspicion is necessary for diagnosing tracheo-bronchial foreign body. Diagnosis is often based on chest radiography. But it has poor sensitivity in picking up small objects. Computed tomography of the chest maybe valuable in identifying small aspirated objects or when associated chest disease is suspected. Bronchoscopy remains the gold standard for diagnosing tracheo-bronchial foreign body. It is frequently both diagnostic and therapeutic. Early detection and removal of foreign body with prompt resuscitation can often be life saving.

\section{Address for correspondence:}

Email:amarabalan@gmail.com

DOI: 10.5530/ijmedph.2.2011.9

\section{CASE 1}

A 78-year-old Caucasian man with history of underlying systemic Hypertension, type 2 diabetes mellitus and coronary artery disease for 18 years was brought to the Emergency Room with sudden onset of breathlessness. He did not have fever, cough or any other symptoms. The person who brought him to the hospital said the patient had pulmonary tuberculosis (TB) 7 years back for which he underwent anti-tuberculous treatment for 6 months.

On examination he was tachypneic and restless. Temperature was 36.8 degree centigrade. Blood pressure was 146/88 mm Hg. Pulse rate was 108/minute regular, of low volume. His cardiovascular and neurological examination was normal. Chest examination revealed decreased intensity of breath sounds in the left hemi thorax. Abdominal examination was unremarkable. Arterial Blood gas analysis in Emergency room (room air) showed $p \mathrm{H}$ of 7.321, $\mathrm{PO}_{2}$ of $62.3 \mathrm{~mm} \mathrm{Hg}, \mathrm{PCO}_{2}$ of 41.2 $\mathrm{mm} \mathrm{Hg}, \mathrm{HCO}_{3}$ of $20.7 \mathrm{mEq} / \mathrm{L}$ and $\mathrm{SaO}_{2}$ of $89.5 \%$ following which the patient was shifted to the Intensive Care Unit (ICU). In the ICU the Arterial blood gas analysis (with high flow oxygen) suggested respiratory acidosis with $p H$ of 7.203, $P O$ of $10^{5} \mathrm{~mm}$ $\mathrm{Hg}, \mathrm{PCO}_{2} \mathrm{~mm} \mathrm{Hg}$ of 50.1, $\mathrm{HCO}_{3}$ of $19 \mathrm{mEq} / \mathrm{L}$ and lactate $6.5 \mathrm{mmol} / \mathrm{L}$. He was then intubated in the ICU.

Routine hematological parameters like hemoglobin, complete blood count, peripheral smear were within normal limits. ESR was $31 \mathrm{~mm} / 1^{\text {st }} \mathrm{hr}$. Random blood glucose was $187 \mathrm{mg} / \mathrm{dl}$. Renal and liver function tests were normal. ECG showed ST depression in lead II, lead III, V5 \& V6. D-dimer was 
250-500.'Troponin T was negative. Chest radiograph revealed haziness in the left upper zone and features suggestive of Pulmonary TB sequelae. CT (computerized tomography) scan showed left sided upper lobe fibro cavity disease possibly the pulmonary TB sequelae. Patient was then planned for a left sided broncho-alveolar lavage (BAL) since haziness was also present in the lower zones. So bedside bronchoscopy was planned in the ICU. As a part of brochoscopic evaluation routine screening of the contralateral normal right lung was done which revealed a rounded green pea in the right lower lobe bronchus. The foreign body was then removed with the help of flexible bronchoscope without any complications. BAL culture and gram stain was negative. Post foreign body removal - ABG showed pH-7.493, PCo2-34.6 mm Hg,
PO2-125 mm Hg, HCo3-26.3 mEq/L \& Lactate-1.4 mmol/L. Patient was then extubated shifted out of ICU. After recovery patient revealed history of aspiration of peanut while eating samosa. Following the removal of the foreign body, the patient continued to do well and was discharged.

\section{CASE 2}

A 45yr old male presented to us with mild respiratory difficulty. He had no other complaints. There was no bistory of any drug intake or atopy. He had no other co-morbidities. He was operated for fracture left humerus one day back in a suburb hospital.

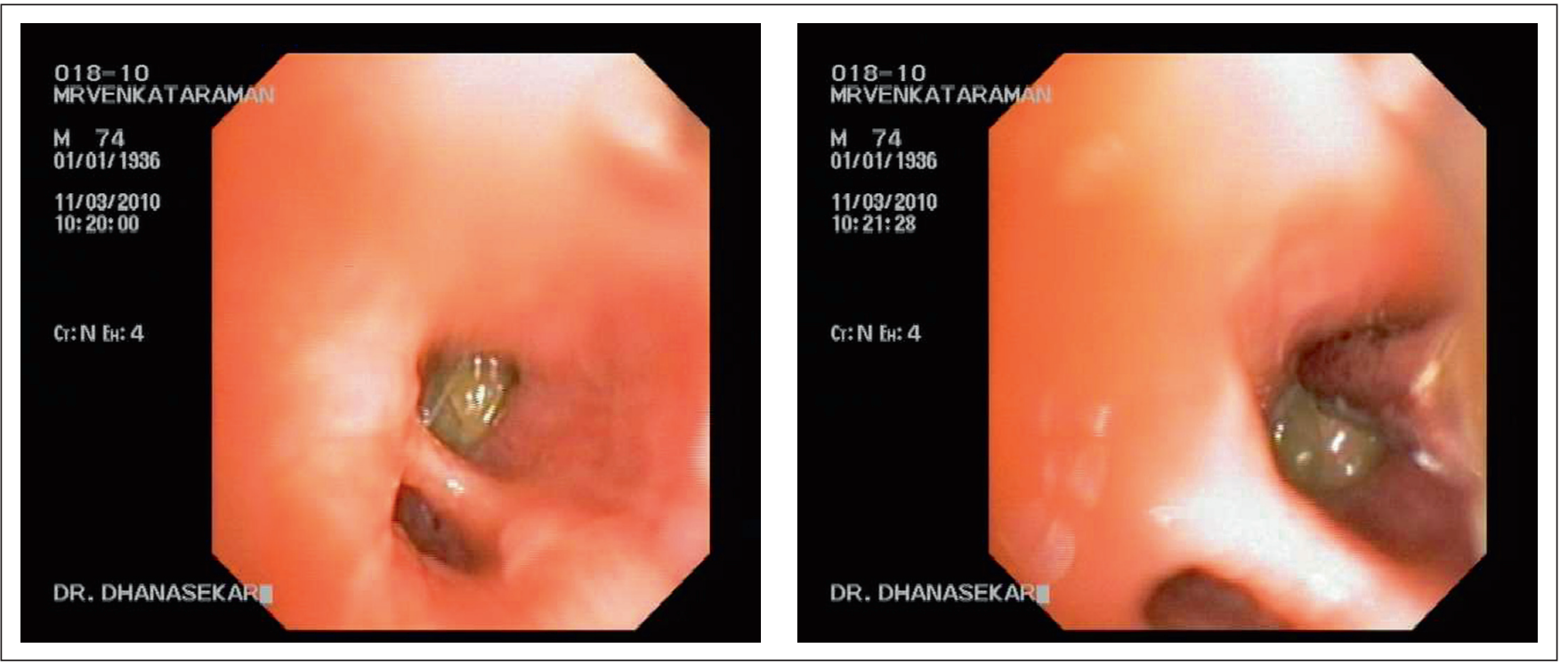

Bronchoscopic View of Foreign body

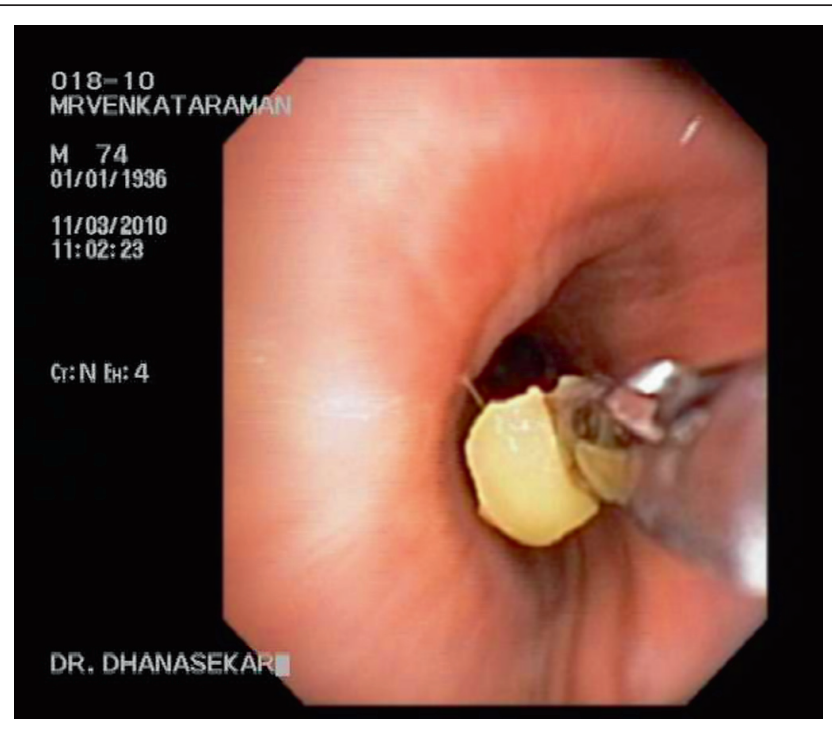

Removal of foreign Body with Bronchoscope

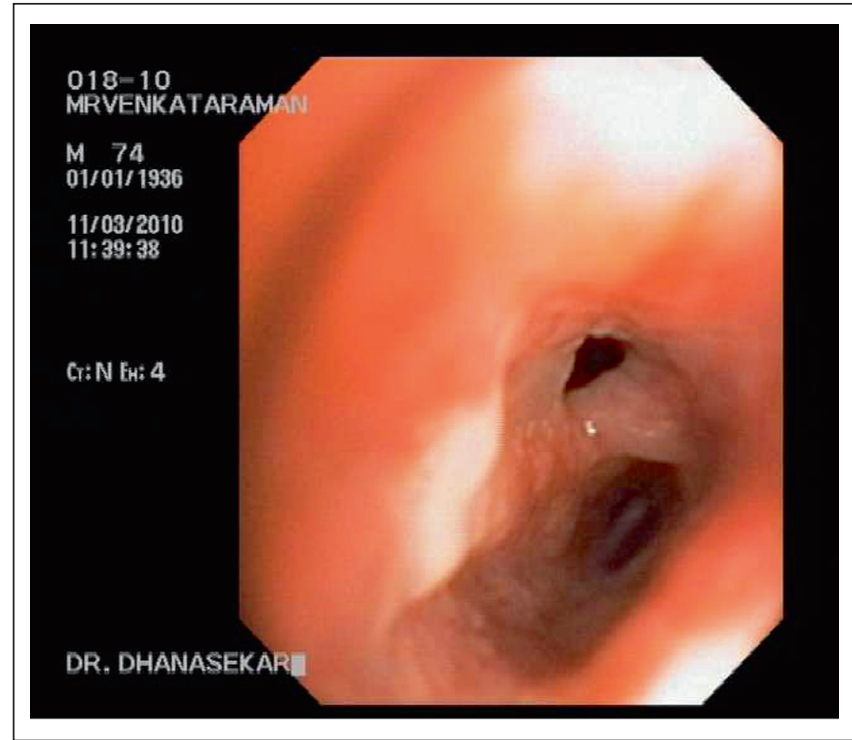

Bronchoscopic View: Post Foreign Body Removal 


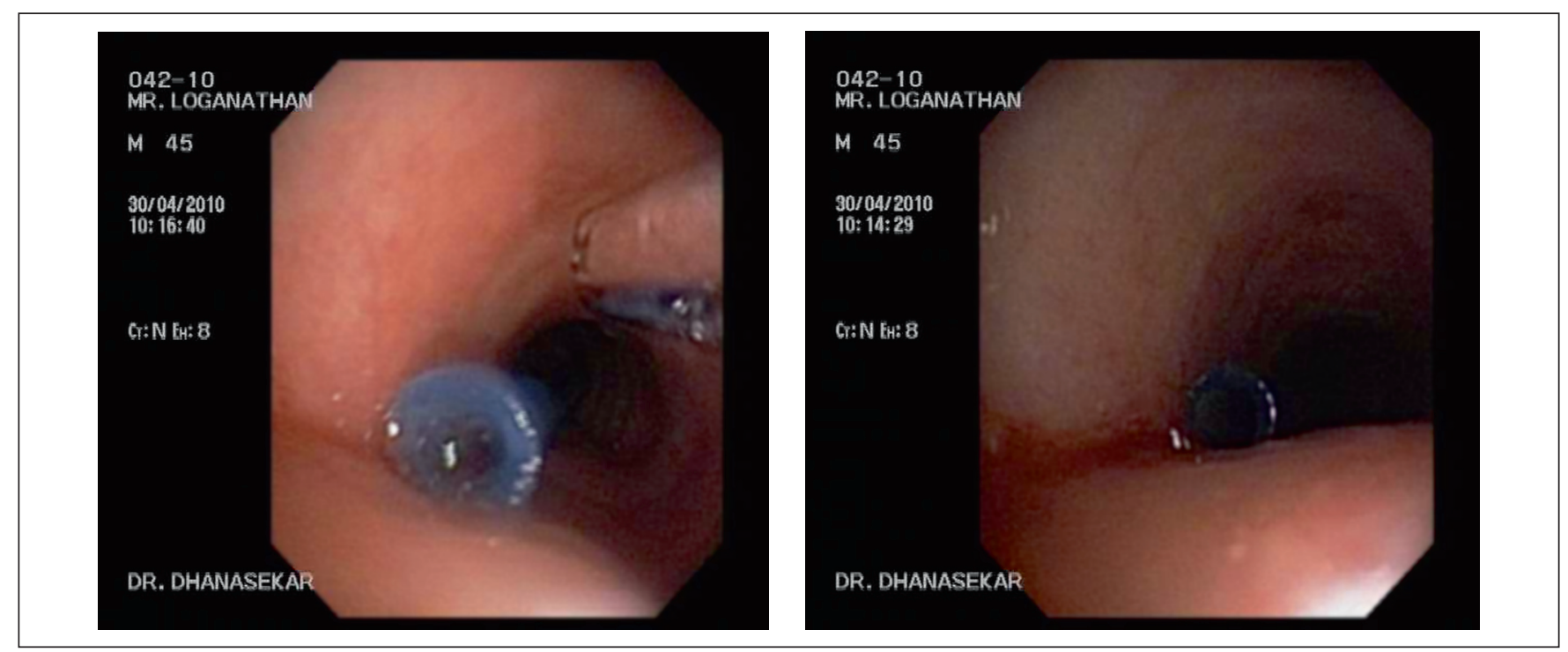

Bronchoscopic View of Foreign Body inside the right main bronchus

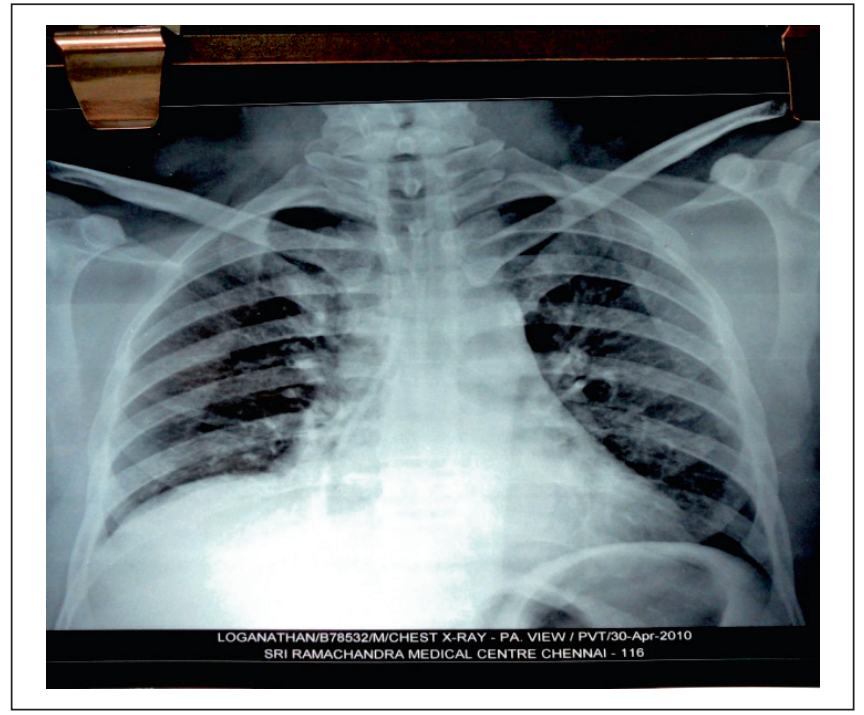

Chest X-ray (PA view) showing the foreign body in the right main bronchus

On physical examination, patient's general condition was fair. His blood pressure was 130/84 mm $\mathrm{Hg}$ and pulse rate was $78 /$ min. Cardiovascular and abdominal examination was unremarkable. Chest examination revealed reduced air entry in to the right lung and chest X-ray posteroanterior (PA) view was advised. Chest radiograph showed a linear opaque foreign body of approximately $6 \mathrm{~cm}$ size lying in the right main bronchus. Then the patient was taken in to the operating room. Under regional anesthesia ( $2 \%$ xylocaine) the foreign body was then removed with flexible bronchoscopy without any complications. The foreign body was a $12 \mathrm{~cm}$ long intubation stylet. Patient was kept under observation for 6 hours and was discharged.

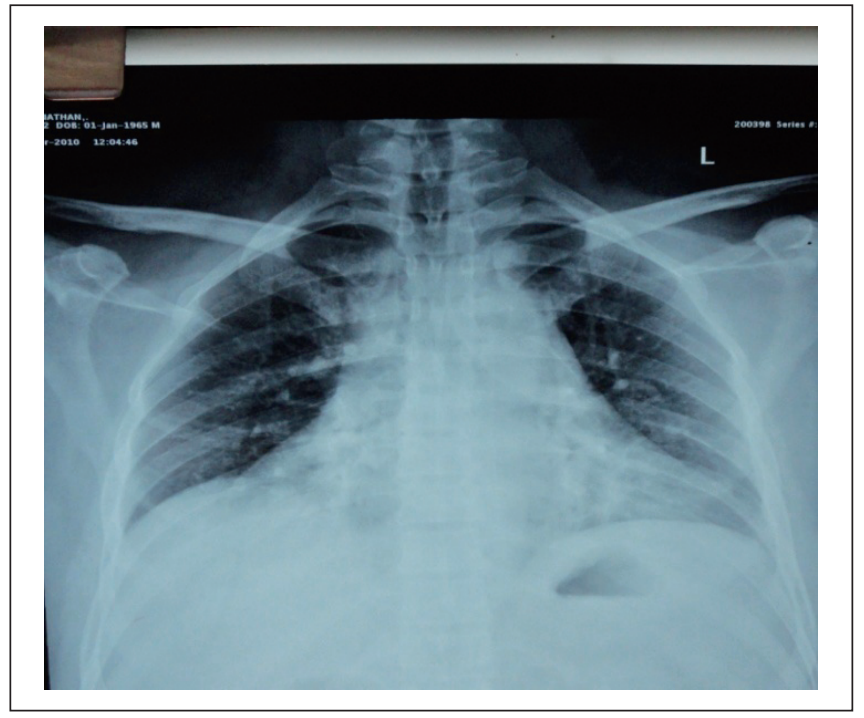

Chest X-ray (PA view) taken after the removal of foreign body

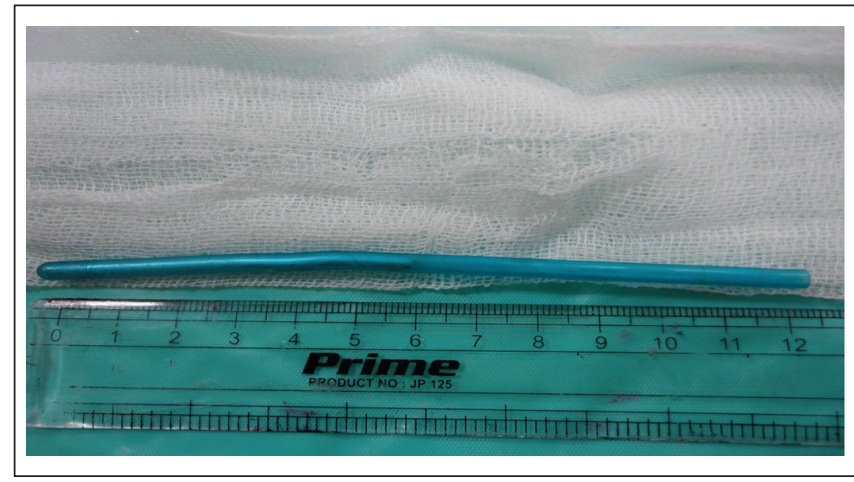

Foreign Body Photograph after Removal 


\section{DISCUSSION}

Foreign bodies can get lodge at any site from supraglottis to the terminal bronchioles. Inhaled foreign bodies are more likely to enter the right bronchial tree than in the left. The commonly encountered foreign bodies are mostly organic $(67 \%)$ compared to inorganic $33 \%$ ) foreign bodies. Organic foreign bodies can cause severe lipoid reaction and result in chemical bronchitis with fever and chest infections. Inorganic Foreign bodies with sharp edges are more fatal compared to those with smooth surface. ${ }^{6}$ Foreign bodies are not always radiopaque and small foreign bodies may cause symptoms without radiographic signs. The diagnostic triad of a trachea-bronchial foreign body include cough, wheezing and decreased breath sounds on the affected side. Other commonly encountered symptoms are cough, fever, breathlessness and wheezing. ${ }^{1}$ Left untreated TFB aspiration can lead to acute upper airway obstruction, laryngeal edema, central obstructive collapse and obstructive emphysema. ${ }^{4}$ Cases of pneumomediastinum or pneumothorax have also been reported. Therefore early recognition and prompt removal of the foreign body is necessary in order to avoid complications. Bronchoscopy is both diagnostic and therapeutic. Flexible bronchoscopy is effective both in the diagnosis and removal of foreign bodies. Flexible bronchoscopy has been demonstrated to be $90 \%$ effective in removal of foreign bodies ${ }^{1}$ and is preferred over rigid bronchoscopy. However, larger aspirates may not be retrievable with a flexible bronchoscope. In those instances use of rigid bronchoscopy must be considered.

In our first case the foreign body was a peanut found in the right lower lobe bronchus. The peculiarity of this case was the unusual mode of presentation. Timely diagnosis was the key in this case. Any delay in the diagnosis and treatment would have proved fatal for this patient.

In our second case the foreign body was rather an unusual one. It was an INTUBATION STYLET. It was found in the right main bronchus. The patient was operated for left humerus fracture in a suburb hospital one day back and he was discharged the next day. After he went home he developed mild respiratory difficulty and went back to the same hospital where they took a chest $\mathrm{x}$-ray posteroanterior view which revealed a linear opaque shadow of $5 \mathrm{~cm}$ in the right main bronchus. They suspected it to be the intubation stylet which must have broken off during extubation after surgery and referred the patient to our tertiary care facility. Repeat chest radiograph done in our centre revealed a linear opaque foreign body of about $5 \mathrm{~cm}$. The foreign body was removed with flexible bronchoscopy and the foreign body turned out to be an intubation stylet. It was the metallic part of the stylet that was visualized in the chest radiograph taken before the procedure. In this case the patient presented with only mild respiratory distress which indicates that adults when compared to children can tolerate foreign body aspiration for a long time.

\section{CONCLUSION}

Tracheobronchial foreign body (TFB) aspiration can be life-threatening. Therefore it is important for clinicians to maintain a high level of suspicion for diagnosing tracheobronchial foreign body. Though incidence is rare in adults its prevalence increases with age. Adults may tolerate aspiration of foreign bodies for a long time without acute life-threatening consequences. ${ }^{3}$ In adults, the fibreoptic bronchoscope is increasingly being used successfully for the removal of foreign bodies. ${ }^{6}$ To prevent delayed diagnosis, characteristic symptoms, signs and radiological findings of foreign body aspiration should be checked in all suspected cases. Bronchoscopy should be considered in patients with an appropriate history. One must proceed for bronchoscopy even when there is a mere suspicion of foreign body. Timely diagnosis and treatment are imperative in order to prevent respiratory sequelae.

\section{REFERENCES}

1. Michael Boyd, Arjun Chatterjee, Caroline Chiles and Robert Chin. Southern Medical Journal, 2009; 102 (2):171-174.

2. Amritpal Singh, Maninder Kaur. Recurrent Pneumonitis due to Tracheobronchial Foreign Body in an Adult. JIACM, 2007; 8(3):242-4.

3. Alia Qureshi, Abdollah Behzadi. Foreign-body aspiration in an adult. Can J Surg, 2008; 51, (3):e69-70.

4. Ganesan S, Raghavan D, Pruthi HS, Borgohain BN. An unusual foreign body in the Bronchus. MJAFI, 2001; (57):161-162.

5. Barthwal MS, Dutta V, Narula T. An Unusual Endobronchial Foreign Body. JAPI 2005; 53:985-987.

6. Bist SS, Saurabh Varshney, Rajesh Kumar, Saxena RK. Neglected Bronchial Foreign Body in an Adult. JK science,2006; 8(4):222-224. 